\title{
Infection Control Practices Associated with Puerperal Sepsis in Harare City Maternity Units
}

\author{
Elizabeth Tirivavi ${ }^{1}$, Violet Chikanya ${ }^{1}$, Paddington Tinashe Mundagowa ${ }^{2, *}$ \\ ${ }^{1}$ College of Health Agriculture and Natural Sciences, Africa University, Mutare, Zimbabwe \\ ${ }^{2}$ The Clinical Research Centre, Africa University, Mutare, Zimbabwe
}

Email address:

tirivavie@africau.edu (E. Tirivavi), chikanyav@africau.edu (V. Chikanya),mundagowap@africau.edu (P. T. Mundagowa)

${ }^{*}$ Corresponding author

\section{To cite this article:}

Elizabeth Tirivavi, Violet Chikanya, Paddington Tinashe Mundagowa. Infection Control Practices Associated with Puerperal Sepsis in Harare City Maternity Units. Central African Journal of Public Health. Vol. 5, No. 1, 2019, pp. 31-35. doi: 10.11648/j.cajph.20190501.15

Received: November 25, 2018; Accepted: December 22, 2018; Published: January 29, 2019

\begin{abstract}
The time of childbirth are responsible for about ten percent of maternal deaths and contribute to severe morbidity as well as long-term disability among many women. Such infections are also termed puerperal sepsis and these are more prevalent in low to medium countries. Puerperal sepsis is preventable by simple and low cost solutions such as handwashing at the time of delivery, damp dusting and use of sterile equipment. The Harare City health department recorded puerperal sepsis as the third cause of maternal mortality, accounting for $18.1 \%$ of all maternal deaths recorded for the year 2016 . The study sought to determine infection prevention and control practices associated with puerperal sepsis in Harare City maternity units. A descriptive cross-sectional research design was employed and data was purposively collected from 84 midwives from 12 polyclinics. Observational walks through visits were carried out to solicit infection control practices and availability of relevant inventory before recording on the checklist. Nurses had above average knowledge on infection and prevention control, however this did not translate to good infection and prevention control practices. The majority of midwives (83\%) had not attend infection prevention and control trainings during the previous year and there was a major challenge of stock-outs of basic consumables and equipment such as surgical gloves and elbow taps.
\end{abstract}

Keywords: Infection, Control, Prevention, Puerperal, Harare

\section{Introduction}

Bacterial infections around the time of childbirth are responsible for about ten percent of maternal deaths and contribute to severe morbidity as well as long-term disability among many women $[1,2,3]$. The most common associated morbidities are chronic pelvic pain, fallopian tube blockage and secondary infertility [4]. Globally, it is estimated that 358 000 women die from the complications of childbirth annually and up to $15 \%$ of these are due to puerperal sepsis [5].

Puerperal sepsis is the infection of the genital tract occurring at any time between the onset of rupture of membranes or labor and the $42^{\text {nd }}$ day postpartum, in which two or more of the following are present: pelvic pain, fever, abnormal vaginal discharge, abnormal smell/foul odor discharge or delay in uterine involution [4]. The condition is easily preventable and is commonly found among the leading causes of maternal mortality in low and medium income countries where resources are scanty $[6,7]$.

About $99 \%$ of deaths from puerperal sepsis in low and middle income countries are preventable with simple, low cost solutions such as healthcare provider hand washing at the time of delivery [5]. Failure to observe recommended Infection Prevention and Control (IPC) in maternity units by both the patients and midwives may result in women developing puerperal sepsis. The provision of sterile instruments, hand hygiene, personal protective clothing and general environmental cleanliness in health facilities can decrease infection rates [7]. Standard infection prevention and control measures such as hand hygiene, use of sterile equipment and proper waste disposal are a cornerstone of peripartum infection prevention [8].

Puerperal sepsis is an important public health problem which is contributing to maternal morbidity and mortality. The world came together in 2015 to intervene and accelerate 
the decline of maternal mortality through the Sustainable Development Goal number 3 (SDG 3). The SDG 3 targets to reduce global mortality ratio to less than 70 per 100000 births [4]. In an effort to reduce infection transmission by health workers and patients, the Center of Disease Control (CDC) drafted the Hand Hygiene guidelines for use in health care settings in 2002. The guidelines provide standard precautions to consider when providing care in clinical settings.

In Africa and Asia, puerperal sepsis (9.7\%) was the second commonest cause of maternal mortality after hemorrhage (11.6\%) [5]. Literature reviewed variations in maternal mortality due to sepsis with Uganda recording 30.9\% and Nigeria 26.3\% [6, 9]. The Zimbabwe Demographic Health Survey of 2016 estimated a maternal mortality rate of 651 per 100000 live births and puerperal sepsis accounted for $10 \%$ of the deaths. An investigation into the causes of maternal mortality in Zimbabwe found out that puerperal sepsis was the third leading cause of maternal mortality accounting for $9 \%$ of the total maternal deaths in 2012 [10].

Harare City health service department was overstretched by an unmatched rapid population growth to available resource ratio which was compromising quality of services [11]. The city health department recorded puerperal sepsis as the third cause of maternal mortality, accounting for $18.1 \%$ of all maternal deaths recorded and there was a significant increase in utilization of the labor facilities due to provision of the Result Based Funds (RBF) (Reproductive health report, 2016, unpublished). The RBF was responsible for payments of all maternity service charges and mothers were not supposed to pay for the services. The increase in utilization of maternity services on the under resourced health system often leads to substandard clinical practices and poor infection control practices [6]. This study sought to determine infection prevention and control practices associated with puerperal sepsis in Harare City maternity units.

\section{Methods}

A descriptive cross-sectional research design was employed in collecting data from Harare City maternity units. The city health department is made up of two infectious disease hospitals, 12 poly clinics, 38 satellite clinics and ten Family Health Service clinics. All the poly clinics were purposively selected for data collection because these facilities offer maternity services. The 2012 national census estimated the Harare City population at 1485231 of which 59.4\% were women of child bearing age and 46488 were expected annual births [12]. Nurse midwives who were working in maternity units and document on policy and infection control guidelines made up the study population.

The sample size for study participants was calculated using the Dobson's formula: $n=Z^{2} \mathrm{pq} / \mathrm{d}^{2}$ were $\mathrm{p}$ was the incidence of puerperal sepsis (9\%) from a study in Zimbabwe [10]. The calculated minimum sample size was 87 and $10 \%$ attrition was expected hence the final sample size was 97 midwives. A total of 12 Maternity Ward managers were also selected as key informants. However, the calculated sample size was not reached due to critical staff shortages and refusal to participate and a total of 84 midwives were interviewed.

Data was collected using pretested interviewer administered questionnaire, key informant interviews and checklists. After consenting to participate, midwives who had worked in the maternity department for at least six months were interviewed. Interviews were carried out in March, 2017 and only those midwives who were available on duty on the day of data collection were interviewed. Observational walks through visits were carried out to solicit infection control practices and availability of relevant inventory before recording on the checklist. Quantitative data were entered and analyzed using Epi-Info version 7 statistical package whilst qualitative data were analyzed manually.

Permission to carry out the study was sought from the Harare Provincial Medical Directorate and ethical clearance was given by Africa University Research Ethics Committee. Informed consents were offered to those who were willing to be interviewed and participation was voluntary.

\section{Results}

Table 1. Socio-demographic characteristics of the study participants.

\begin{tabular}{lll}
\hline Variable & Frequency & Percentage \\
\hline Sex & & \\
Male & 4 & 5 \\
Women & 80 & 95 \\
Age & & \\
$34-43$ & 36 & 43 \\
$44-53$ & 28 & 33 \\
$>53$ & 20 & 24 \\
Length in Service (years) & & \\
$<10$ & 21 & 25 \\
$10-14$ & 41 & 49 \\
$>14$ & 22 & 26 \\
\hline
\end{tabular}

The Table 1 above depicts the demographic data of study participants. Eighty (95.2\%) midwives interviewed were females and age range of the participants was 34-64 years with a mean age of 45 years. Three quarters of the midwives had more than ten years working experience.

Regarding definition of puerperal sepsis, 76 (91\%) mentioned that it was infection of the perineum during delivery and the six weeks in the postnatal period. Only 20 (24\%) mentioned that it was general body illness in the postnatal period. When asked to state how they detected postpartum infections, $45(54 \%)$ would suspect infection if the temperature is more than $38^{\circ} \mathrm{C}, 69(82 \%)$ mentioned the presence of pain and redness on the perineum and a foul smelling vaginal discharge. Only $7(8 \%)$ would suspect infection if there is poor involution within the first eight days of puerperium.

Most nurses were able to state at least more than three signs and symptoms of infections, (sepsis) and demonstrated above average knowledge in diagnosis of postpartum infections. Pyrexia and pain were the main determining factors for nurses to suspect post-delivery infections. With 
reference to management of postpartum infections, 71(84\%) participants would give antibiotics according to protocol whilst $15(18 \%)$ would give intravenous fluids and antipyretics in addition to antibiotics. More than half of the participants would use more than one treatment modality and were more likely to refer for further management to prevent complications. When nurses were asked to state measures they would take to prevent infection, the majority 78 (93\%) mentioned use of sterile gloves and sterile delivery packs during delivery and $74(88 \%)$ would avoid frequent vaginal examination.

Sixty (71\%) participants mentioned unhygienic delivery procedures and retained products of conception as major predisposing factors to postpartum infections. However, $67(80 \%)$ mentioned frequent vaginal examination, and $27(32 \%)$ cited premature rapture of membranes as most common causes of postpartum infections. Most nurses $64(76 \%)$ had above average knowledge as they were able to mention at least more than thee common causes of postpartum infection. Table 2 below illustrates the advice and corresponding number of midwives who mentioned that they would counsel a woman with regard to puerperal sepsis prevention.

Table 2. Advice and corresponding number of midwives who mentioned that they would counsel a woman with regard to puerperal sepsis prevention.

\begin{tabular}{lll}
\hline Responses & Frequencies $(\mathbf{n}=\mathbf{8 4})$ & Percentage \\
\hline Good personal hygiene & 81 & 91 \\
Frequent change of pads & 79 & 94 \\
Visit hospital if mothers suspects retained products & 78 & 93 \\
Frequent vulva cleaning & 66 & 79 \\
Using cold sitz baths & 28 & 33 \\
\hline
\end{tabular}

Majority of the participants $(93 \%)$ agreed that they had an IPC focal person at their unit. More than three quarters of the participants $72(86 \%)$ mentioned that they have infection control committees. About $83 \%$ of the interviewed midwives had not attended a workshop on IPC during the past year. The most common personal protective clothing were aprons, gloves, face masks, goggles and boots. However the supply for these protective clothing was erratic and 36\% mentioned that the protective clothing were uncomfortable. Aprons were washed two to three times a week and the maternity units in Harare City used sodium hypo-chlorite, betadine and methylated spirit as disinfectants/antiseptics. Maternity floors were cleaned per rising need and delivery instruments were cleaned and autoclaved after every procedure.

Table 3. Attitudes of Health care workers on infection prevention and control of Puerperal sepsis in maternity units.

\begin{tabular}{|c|c|c|c|c|c|}
\hline Attitude questions on infection control practices related to puerperal sepsis & $\begin{array}{l}\text { Strongly } \\
\text { agree }\end{array}$ & Agree & Neutral & Disagree & $\begin{array}{l}\text { Strongly } \\
\text { disagree }\end{array}$ \\
\hline Sepsis in the puerperium can be prevented/detected earlier & $95 \%$ & $5 \%$ & - & - & - \\
\hline Damp dusting is a very important measure in preventing infection & $91.7 \%$ & $5.9 \%$ & $2.4 \%$ & - & - \\
\hline Washing of hands is very important before and after attending a patient & $65.5 \%$ & $18.8 \%$ & $15.7 \%$ & - & - \\
\hline Following standard precautions is your responsibility & $88.6 \%$ & $6.9 \%$ & $10.5 \%$ & - & - \\
\hline $\begin{array}{l}\text { Use of gloves while attending a patient is a useful strategy for reducing risk of } \\
\text { transmission of organisms }\end{array}$ & $52.5 \%$ & $29.6 \%$ & $12.7 \%$ & $3.1 \%$ & $2.1 \%$ \\
\hline Frequent vaginal examinations can lead to development of puerperal sepsis & $82 \%$ & $6.4 \%$ & $11.6 \%$ & - & - \\
\hline
\end{tabular}

The Table 3 above shows the participants' attitudes towards IPC for prevention of puerperal sepsis in the maternity units. Half of the sampled clinics had wash basins with elbow/knee taps as well as soap dispensers and liquid soap was always available at $90 \%$ of the clinics. All clinics did not have an IPC policy although IPC guidelines were available at $70 \%$ of the clinics. Sterile delivery packs were available at all units but only eight clinics had functional autoclaves. Regarding protective equipment, almost all health facilities (90\%) had sterile gloves available all the times, $80 \%$ had head covers available in labor wards and face masks were available in $75 \%$ of the facilities.

\section{Discussion}

The aim of the study was to assess the infection prevention and control practices associated with puerperal sepsis in Harare City maternity units. Almost $95 \%$ of interviewed midwives were females. This was mainly due to the nursing profession being dominated by females [13]. Three quarters of the participants had a working experience of more than 10 years and this added to maturity and better decision making [14].

Nurses had above average knowledge on infection and prevention control, however this did not translate to good infection and prevention control practices observed during the walk through visits. This finding was consistent with a Palestinian study in which $98 \%$ of the trained nurses had knowledge on prevention of nosocomial infections but only $78 \%$ of the participants practiced prevention of the hospital acquired infections [15]. The major hindrances to practice were cited as lack of equipment, poor working environment and conditions as well as critical staff shortages.

Given the importance of handwashing, it was noted with concern that almost $16 \%$ of midwives disagreed that washing of hands was very important before and after attending a patient. A study in Kenyan found out that although staff routinely washed their hands before and after procedures, only $75 \%$ did vigorous rubbing of the hand before conducting a procedure deemed to be aseptic (16). In India, 
adherence to recommended hand-washing practices remained unacceptably low, rarely exceeding $40 \%$ of situations in which hand hygiene was indicated [17]. Hand hygiene is considered a very crucial standard precaution and thus, should be adhered to at all times in order to prevent and control the proliferation of infectious microbes in the maternity units.

Infection control guidelines recommend that nurses should be sensitized through trainings at least twice a year [18]. Continuous training aiming at knowledge improvement on the use of appropriate and effective methods of disinfection and sterilization could reduce the frequency of women developing hospital acquired maternal infections. The majority of midwives in Harare City did not attend any infection and prevention control training during the past year preceding the study. Attending workshops on updating skills on infection prevention and control, had the potential of improving the quality of obstetric care in maternity units [19].

This study revealed that reasons for not using personal protective equipment was due to stock outs and lack of comfort of the equipment used. Participants cited use of uncomfortable protective equipment slowing down their working rate in an unbalanced nurse to patient ratio environment. This was similar to findings in an Indian study where patient overcrowding had negative effects on infection prevention and control efforts [6]. The failure of the staff and environment to cope with the number of clients to be served contributes to poor quality of health services [20].

It was also found out that only half of the sampled facilities had wash basins with elbow or knee taps. This finding concurred with a Nigerian study in which a third of the observed facilities had no 'hands-free' taps [21]. It was encouraging to note that liquid soap or handwashing and sterile gloves were reported to be widely available at the majority of the facilities which was consistent with a study in Pakistan [16].

\section{Conclusion}

The study revealed that majority of the midwives did not implement the recommended universal infection control practices. Many inadequacies stemmed from lack of necessary protective equipment supplies and infection control policy for Harare City Health Department. However even when the resources were available they were often inconsistently used. The chronic staff shortages also compromised the infection control practices. Limited participation by a majority of nurses in continuing education sessions on infection related issues contributed to compromised quality of care. An improvement in supply of personal protective equipment and supporting infrastructure such as elbow taps has the potential of improving infection control practices in Harare City maternity units.

Generally city health clinics have critical staff shortages and the situation was worsened when some health workers were not on duty either as a result of illness or had to be excluded due to other commitments and/ or dropped because they were extremely busy with patients. However, to alleviate this, participants were given assurance that the study results will benefit them and the patients under their care. The study also excluded private facilities which offered maternity services in the capital.

\section{Author Contributions}

ET came up with the concept, wrote the protocol, collected data and PTM wrote the manuscript. PTM and VC reviewed the protocol and the manuscript.

\section{Acknowledgements}

We would like thank the Harare Provincial Medical Director for permission to undertake this study. We are also thankful to Harare city staff members and mothers for their participation in the study.

\section{References}

[1] Say L, Chou D, Gemmill A, Tuncalp O, Moller AB, Daniels J, et al. Global causes of maternal death: a WHO systematic analysis. Lancet Glob Health. 2014; 2(6):e323-33.

[2] Khan KS, Wojdyla D, Say L, Gulmezoglu AM, Van Look PF. WHO analysis of causes of. Maternal death: a systematic review. Lancet. 2006; 367(9516):1066-74.

[3] Shamshad, Shamsher, S and Rauf B. (2010). Puerperal Sepsis Still a Major Threat For.Parturient Department of Gynecology, Khyber Girls Medical College, Peshawar, Pakistan.

[4] World Health Organization. (2015). Recommendations for Prevention and Treatment of Maternal Peripartum Infections, Highlights and Key Messages from the World Health Organization: Global Recommendations. WHO Press, Geneva.

[5] Hussein J. \& Walker L. (2012). Puerperal sepsis in Low - and middle income settings: Past, present and future. Royal college of Obstetricians and Gynecologists.

[6] Khaskheli, M., Baloch, S., \& Sheeba A. (2013).Risk factors and complications of puerperal sepsis at a tertiary healthcare center

[7] Hussein J, Mavalankar D. V, Sharma S and D'Ambruoso L. (2011). A review of health system infection control measures in developing countries: what can be learned to reduce maternal mortality

[8] World Health Organization (2015) Trends in Maternal Mortality 1990-2015: Geneva, Switzerland.

[9] Ngonzi, J. ,Yarine, F. T. ,Mukasa, P. K.,Salongo, W., Kabakyenga, J., Sezalio, W., Wouters, K., Yves Jacquey, Y. \& Geertruyde J.(2016). Puerperal sepsis, the leading cause of maternal deaths at a Tertiary University Teaching Hospital in Uganda.

[10] Mlambo, C, Chinamo C, \& Zingwe T. (2013). An Investigation of the Causes of Maternal Mortality in Zimbabwe: Harare Zimbabwe. 
[11] Harare 24. (2014, August 9). Harare 24. Retrieved from Poor Health services force expectant mothers to turn to Vapositori: Available at: http://harare24.com/index-id-news-zk22333.html.

[12] UNICEF. (2014). Zimbabwe 2012 Population census results, Harare Province. Harare, Zimbabwe: UNICEF \& ZIMSTAT.

[13] Herakova, L. (2012). Nursing masculinity: Male nurses' experiences through a co-cultural lens. Howard Journal of Communications, 23(4):332-350.

[14] Kayombo, E. J. (2013). Impact of Training traditional birth attendants on maternal mortality and morbidity in SubSaharan African countries. Tanzania Journal of Health Research Volume 15, Number 2.

[15] Fashafsheh I, Ayed A, Eqtait F, Harazneh L (2015) Knowledge and Practice of Nursing Staff towards Infection Control Measures in the Palestinian Nursing Department, Arab American University: Palestine Journal of Education and Practice (Online) Vol.6, No.4.

[16] Mgongo (2010). Post caesarean section wound sepsis at Mnazi Mmoja Hospital: University of Nairobi.
[17] Mathur, P. (2011). Hand hygiene: Back to the basics of infection control. Retrieved from https://www.ncbi.nlm.nih.gov/pmc/articles/PMC3249958/.

[18] Centers for Disease Control and Prevention. (2012). Hand hygiene basics.http://www.cdc.gov/handhygiene/Basics.html.

[19] Sarani, H., Balouchi, A., Masinaeinezhad, N. \& Ebrahimitabs E. (2014). Knowledge, Attitude and Practice of Nurses about Standard Precautions for Hospital-Acquired Infection in Teaching Hospitals Affiliated to Zabol University of Medical Sciences.

[20] Yakob E., Lamaro, T and Henok A. (2015). Knowledge, Attitude and Practice towards Infection Control Measures among Mizan-Aman General Hospital Workers, South West Ethiopia. Department of Nursing, Mizan-Tepi University, Mizan, Ethiopia

[21] Friday, O., Edoja, I. O., Osasu, A., Chinenye, N., Cyril, M., Lovney, K. \& Julia H. (2012).Assessment of infection control practices in maternity units in Southern Nigeria. International Journal for Quality in Health Care; Volume 24, Number 6: pp. 634-640. 